\title{
Charge collection in irradiated HV-CMOS detectors
}

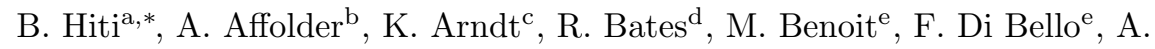

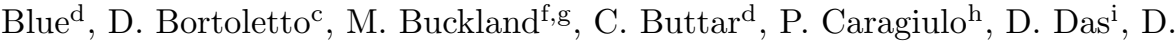
Doering $^{\mathrm{h}}$, J. Dopke ${ }^{\mathrm{i}}$, A. Dragone ${ }^{\mathrm{h}}$, F. Ehrler ${ }^{\mathrm{j}}$, V. Fadeyev ${ }^{\mathrm{b}}$, W. Fedorkok ${ }^{\mathrm{k}}$ Z . Galloway $^{\mathrm{b}}$, C. Gay ${ }^{\mathrm{k}}$, H. Grabas ${ }^{\mathrm{b}}$, I. M. Gregor ${ }^{\mathrm{l}}$, P. Grenier ${ }^{\mathrm{h}}$, A. Grillo ${ }^{\mathrm{b}}$, Y. $\operatorname{Han}^{\mathrm{m}}$, M. Hoeferkamp ${ }^{\mathrm{n}}$, L. B. A. Hommels ${ }^{\mathrm{o}}$, T. Huffman ${ }^{\mathrm{c}}$, J. John ${ }^{\mathrm{c}}, \mathrm{K}$. Kanisauskas $^{\mathrm{c}, \mathrm{d}}$, C. Kenney ${ }^{\mathrm{h}}$, G. Kramberger ${ }^{\mathrm{a}}$, Z. Liang ${ }^{\mathrm{b}}$, I. Mandić ${ }^{\mathrm{a}}$, D. Maneuski $^{\mathrm{d}}$, F. Martinez-Mckinney ${ }^{\mathrm{b}}$, S. McMahon ${ }^{\mathrm{c}, \mathrm{i}}$, L. Meng $^{\mathrm{f}, 1}$, M. Mikuž ${ }^{\mathrm{a}, \mathrm{p}}$, D. Muenstermann ${ }^{\mathrm{q}}$, R. Nickerson ${ }^{\mathrm{c}}$, I. Peric ${ }^{\mathrm{j}}$, P. Phillips ${ }^{\mathrm{c}, \mathrm{i}}$, R. Plackett ${ }^{\mathrm{c}}$, F.

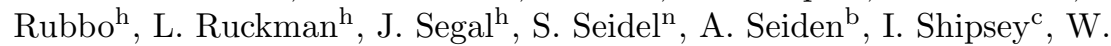
Song $^{\mathrm{m}}$, M. Stanitzki ${ }^{1}$, D. Su ${ }^{\mathrm{h}}$, C. Tamma ${ }^{\mathrm{h}}$, R. Turchetta ${ }^{\mathrm{i}}$, L. Vigani ${ }^{\mathrm{c}}$, J. Volk $^{\mathrm{b}}$, R. Wang ${ }^{\mathrm{r}}$, M. Warren ${ }^{\mathrm{s}}$, F. Wilson ${ }^{\mathrm{i}}$, S. Worm ${ }^{\mathrm{t}}$, Q. Xiu ${ }^{\mathrm{m}}$, J. Zhang ${ }^{\mathrm{r}}, \mathrm{H} . \mathrm{Zhu}^{\mathrm{m}}$

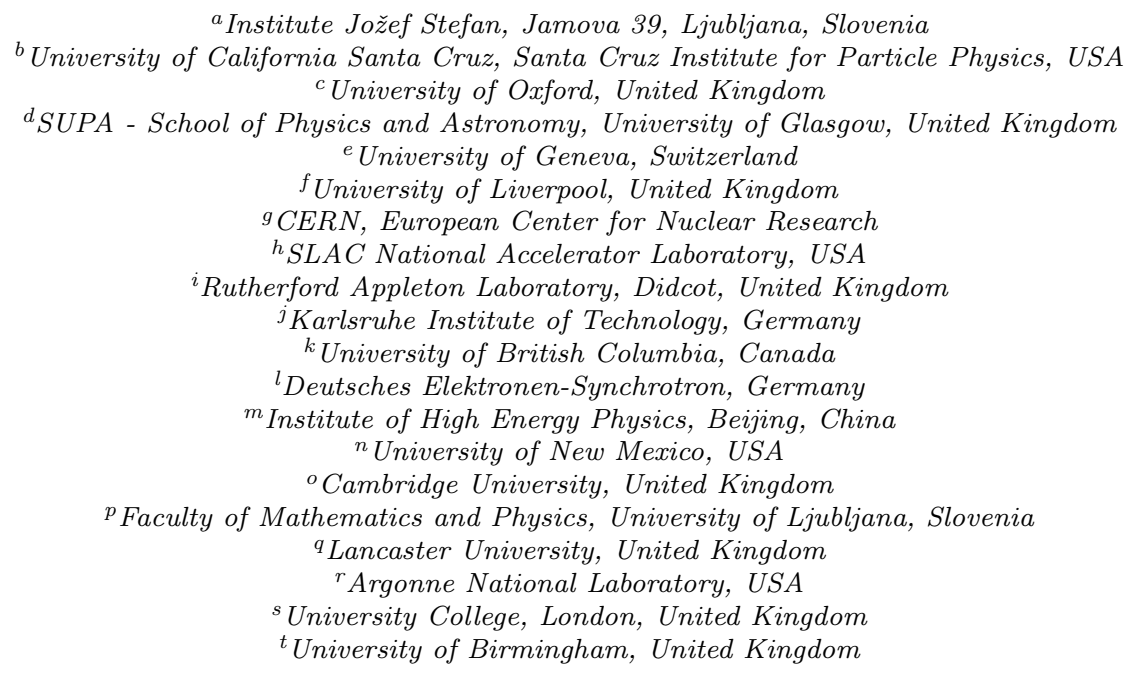

\begin{abstract}
Active silicon detectors built on p-type substrate are a promising technological solution for large area silicon trackers such as those at the High Luminosity LHC, but the radiation hardness of this novel approach has to be evaluated. Active n-in-p strip detector prototypes CHESS2 for ATLAS with different substrate
\end{abstract}

\footnotetext{
*Corresponding author.

Email address: bojan.hiti@ijs.si (B. Hiti)
} 
resistivities in the range of $20-1000 \Omega \mathrm{cm}$ were irradiated with neutrons and protons up to a fluence of $2 \times 10^{15} \mathrm{n}_{\mathrm{eq}} \mathrm{cm}^{-2}$ and $3.6 \times 10^{15} \mathrm{n}_{\mathrm{eq}} \mathrm{cm}^{-2}$. Charge collection in passive test structures on the chip was evaluated using Edge-TCT and minimum ionising electrons from ${ }^{90} \mathrm{Sr}$. Results were used to assess radiation hardness of the detector in the given fluence range and to determine parameters of initial acceptor removal in different substrates.

Keywords: Active silicon detectors; Charge collection efficiency; LHC upgrade

\section{Introduction}

The High Luminosity LHC (HL-LHC) upgrade is foreseen to reach the instantaneous luminosity up to $5 \times 10^{34} \mathrm{~cm}^{-2} \mathrm{~s}^{-1}$, resulting in up to 200 protonproton collisions within individual bunch crossings [1]. Since the existing Inner

5 Detector of the ATLAS experiment [2] is not designed for the resulting readout rate and radiation damage it will have to be replaced in the Phase II Upgrade [3. One technological option for the new detector is to use silicon sensors with CMOS readout electronics implemented on the same chip. To ensure sufficient radiation hardness and time resolution, charge collection has to rely on the drift 10 of charge carriers in depletion zone. Therefore, the detector has to be designed to allow usage of sufficiently high bias voltages.

One of the CMOS technologies that allow high bias voltages is called HVCMOS. The advantage of HV-CMOS detectors compared to traditional hybrid silicon modules is a possibility of a greater segmentation with a corresponding improvement in tracking resolution. In addition, the possibility to produce detectors in commercially available CMOS processes on industrial grade silicon wafers offered by a large number of vendors allows significantly simplified and faster production.

Depleted CMOS detectors are produced on p-type substrates, with a typical resistivity of 10 to a few $1000 \Omega \mathrm{cm}$, and are usually operated under partial depletion. Numerous studies such as [4] have proved functionality of HVCMOS detectors after irradiation to $2 \times 10^{15} \mathrm{n}_{\mathrm{eq}} \mathrm{cm}^{-2}$, expected for the outer 
layers of the ATLAS pixel detector at the HL-LHC. It was found that radiation induced removal of initial acceptors is an important effect in these substrates [12. As a result a deeper depletion with more available charge can be achieved, leading to a better performance and a potentially longer detector lifetime.

HV-CMOS technology has been investigated as an option for the strip detector at the upgraded ATLAS experiment. A prototype chip named CHESS2 (CMOS HV/HR Evaluation for Strip Sensors) was produced for this purpose.

so This paper reports on effects of irradiation on charge collection in this detector.

\section{Sample and irradiation}

The CHESS2 chip 13 has been produced by AMS in a $350 \mathrm{~nm}$ HV-CMOS process. The chip size is $18.6 \mathrm{~mm} \times 24.3 \mathrm{~mm}$ and consists of three strip arrays with digital signal encoding and several test structures for evaluating analogue and digital functionality of the chip, shown in Figure1. Measurements presented in this work were made with two passive test structures (diodes), a $3 \times 3$ n-inp pixel array with a pixel size of $630 \mu \mathrm{m} \times 40 \mu \mathrm{m}$ for edge transient current technique (Edge-TCT) and a large (pad-like) array of interconnected implants with a total size of $1260 \mu \mathrm{m} \times 1280 \mu \mathrm{m}$ for measurements with ${ }^{90} \mathrm{Sr}$. The fill factor of collecting electrodes in the pixel is $53 \%$ for both structures. CHESS2 chips are produced on four different p-type substrates with initial resistivities of 20 , 50-100 (50), 200-300 (200), and 600-2000 (1000) $\Omega \mathrm{cm}$. Values in parentheses are used in the rest of the text. Chips are thinned to $250 \mu \mathrm{m}$ without further back plane processing. High voltage of up to $120 \mathrm{~V}$ for reverse biasing of the 45 sensor is applied between the collecting n-type electrodes and a p-type bias rail surrounding each pixel.

The samples were irradiated with reactor neutrons in Jožef Stefan Institute's TRIGA reactor [14, and with protons (Figure 1) at the CERN PS IRRAD facility $(24 \mathrm{GeV} / c$, beam FWHM $14 \mathrm{~mm})$ 15] and at Los Alamos Neutron Science Centre - LANSCE $(800 \mathrm{MeV} / c$, beam FWHM $12 \mathrm{~mm})$ up to maximal fluences of $2 \times 10^{15} \mathrm{n}_{\mathrm{eq}} \mathrm{cm}^{-2}$ and $3.6 \times 10^{15} \mathrm{n}_{\mathrm{eq}} \mathrm{cm}^{-2}$ for neutrons and protons respec- 


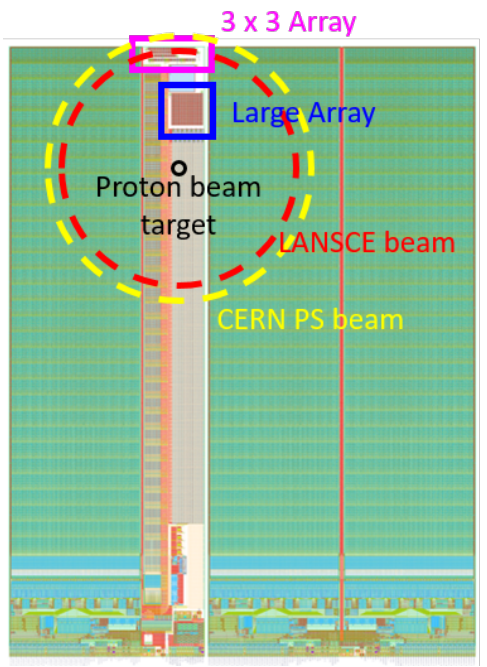

Figure 1: CHESS2 sample with test structures. Position and size $(1 \sigma)$ of the beams during proton irradiation are also shown.

tively. The irradiation summary is shown in Table 1 . The proton irradiated chips were not irradiated uniformly so certain test structures may have received a lower local fluence than listed in Table 1, I-V measurements indicate that some test structures irradiated at LANSCE have an order of magnitude smaller leakage current than PS irradiated samples, implying that the local fluence in these structures is lower than nominal.

Measurements with all irradiated samples were carried out before and after annealing at $60^{\circ} \mathrm{C}$ for 80 minutes. Annealing had no measurable effect on

60 Edge-TCT measurements, whereas the amount of collected charge increased by $\approx 10 \%$ in annealed samples.

\section{Edge-TCT measurements}

In Edge-TCT a narrow laser beam $(\mathrm{FWHM}=10 \mu \mathrm{m})$ is directed to the edge of the detector mounted on high precision moving stages. More details about this technique can be found in [16. By measuring the response of the detector to a laser pulse at different depths the dimension of the depletion region can 


\begin{tabular}{l|c|c|c|c|} 
& $\Phi_{\text {eq }}(20 \Omega \mathrm{cm})$ & $\Phi_{\text {eq }}(50 \Omega \mathrm{cm})$ & $\Phi_{\text {eq }}(200 \Omega \mathrm{cm})$ & $\Phi_{\text {eq }}(1000 \Omega \mathrm{cm})$ \\
\hline PS (p) & $4.2,8.7$ & $\mathrm{n} / \mathrm{a}$ & $4.2,8.7$ & $4.2,8.7$ \\
\hline LANSCE (p) & 7.8 & $7.8,14,36$ & $7.8,14,36$ & 7.8 \\
\hline Ljubljana (n) & \multicolumn{4}{|c|}{$1,3,5,10,20$} \\
\hline
\end{tabular}

Table 1: Irradiated samples by fluence and initial resistivity. All fluences are given in $10^{14} \mathrm{n}_{\mathrm{eq}} \mathrm{cm}^{-2}$.

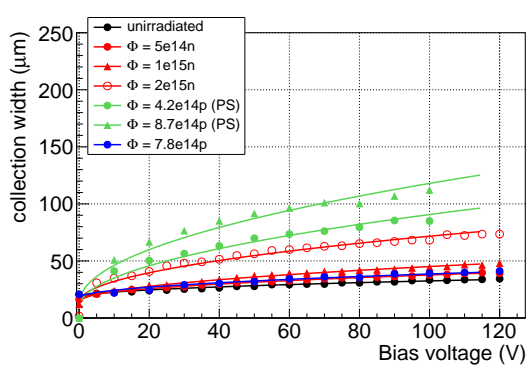

(a) $20 \Omega \mathrm{cm}$

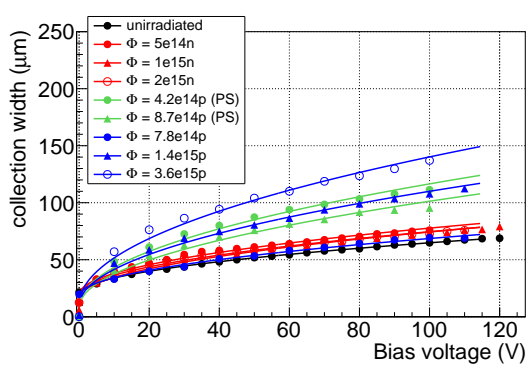

(c) $200 \Omega \mathrm{cm}$

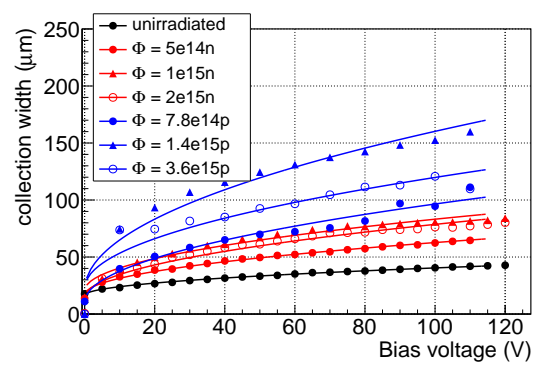

(b) $50 \Omega \mathrm{cm}$

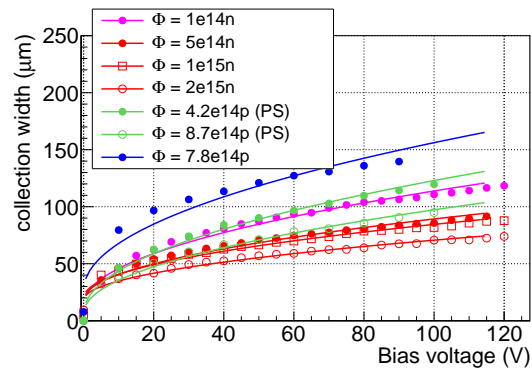

(d) $1000 \Omega \mathrm{cm}$

Figure 2: Dependence of depletion depth on bias voltage for different fluences and initial wafer resistivities. 
be estimated (see [16]). One dimensional Edge-TCT scans were made along the sample depth at the centre of the long side of the test structure. Depletion depth was evaluated as FWHM of resulting charge collection profiles. The dependence of depletion depth on bias voltage is shown in Figure 2 for different fluences and initial wafer resistivities. In $20 \Omega \mathrm{cm}$ samples, before irradiation a depletion depth of $30 \mu \mathrm{m}$ can be achieved. After neutron irradiation the depletion depth gradually increases due to acceptor removal [12] and reaches $70 \mu \mathrm{m}$ after a fluence of $2 \times 10^{15} \mathrm{n}_{\mathrm{eq}} \mathrm{cm}^{-2}$. After proton irradiation (PS) a substantially larger depletion depth of $80 \mu \mathrm{m}$ is observed already after $4.2 \times 10^{14} \mathrm{n}_{\mathrm{eq}} \mathrm{cm}^{-2}$ and a maximal depth of $120 \mu \mathrm{m}$ is observed after $8.7 \times 10^{14} \mathrm{n}_{\mathrm{eq}} \mathrm{cm}^{-2}$. However, the increase of the depletion depth in the sample irradiated at LANSCE to $7.8 \times 10^{14} \mathrm{n}_{\mathrm{eq}} \mathrm{cm}^{-2}$ is only marginal, but the received fluence is probably overestimated as discussed in Section 2. Increased depletion depth after irradiation was also measured on wafers with the initial resistivity of 50 and $200 \Omega \mathrm{cm}$. Due to the higher substrate resistivity the depletion depth in these wafers is larger and in the $200 \Omega \mathrm{cm}$ wafer exceeds $70 \mu \mathrm{m}$ at all fluences. In the $1000 \Omega \mathrm{cm}$ sample depletion depth could not be measured before irradiation because of an electrical breakdown. In irradiated chips of this resistivity the depletion depth decreases from $120 \mu \mathrm{m}$ after $1 \times 10^{14} \mathrm{n}_{\mathrm{eq}} \mathrm{cm}^{-2}$ to $70 \mu \mathrm{m}$ with increasing fluence, indicating that the effects of acceptor removal, if any, would be observed at lower fluences. For all initial resistivities the depletion depth is significantly larger after irradiation with protons than with neutrons at comparable fluences.

The data in Figure 2 were fitted with the function

$$
W_{\mathrm{depl}}=w_{0}+\sqrt{\frac{2 \varepsilon}{e_{0} N_{\mathrm{eff}}} V_{\mathrm{bias}}},
$$

90 the effective space charge concentration. The square root dependence comes from the abrupt junction, constant space charge approximation. An additional term $w_{0}$ describes effects of built in voltage and finite beam width to account for the observed offset at zero volts. The dependence of fitted values of $N_{\text {eff }}$ on 
evolution of $N_{\text {eff }}$ can be observed. The effective space charge concentration initially decreases due to acceptor removal. At higher fluences after the initial acceptors are removed the space charge concentration increases linearly due to radiation induced deep acceptors. The fluence at which the process of initial acceptor removal is finished has been empirically found to depend on the initial resistivity of the material - removal in low resistivity material generally occurs at higher fluences. The behaviour of $N_{\text {eff }}$ is described by the function [17:

$$
N_{\text {eff }}=N_{\text {effo }}-N_{\mathrm{c}} \cdot\left(1-\exp \left(-c \cdot \Phi_{\text {eq }}\right)\right)+g_{\mathrm{C}} \cdot \Phi_{\text {eq }},
$$

where $N_{\text {effo }}$ is the initial doping concentration, $N_{\mathrm{c}}$ is the concentration of acceptors that are removed, $c$ is the acceptor removal constant describing the characteristic fluence for the removal, and $g_{\mathrm{C}}$ is stable damage introduction rate. The function was fitted to the neutron irradiation data and the fit results are shown in Table 2. For the 20,50 and $200 \Omega \mathrm{cm}$ samples the factor $g_{\mathrm{C}}$ was fixed to the value of $0.02 \mathrm{~cm}^{-1}[18,19]$. The fitted acceptor removal constants $c$ are shown in Figure 4 and are compared with other CMOS detectors [7-10]. Given that the acceptor removal is generally faster in less doped material, the values of $c$ for CHESS2 are lower than expected, especially for the $200 \Omega \mathrm{cm}$ material. However, the results are compatible with measurements on the H35Demo chips produced by the same manufacturer [9]. The measurements with the $1000 \Omega \mathrm{cm}$ sample were fitted with $N_{\text {eff }}=N_{\text {effo }}+g_{\mathrm{C}} \cdot \Phi_{\text {eq }}$ with $N_{\text {effo }}$ and $g_{\mathrm{C}}$ as free parameters. The resulting value of $g_{\mathrm{C}}=(0.020 \pm 0.003) \mathrm{cm}^{-1}$ agrees with literature.

The values of $N_{\text {eff }}$ measured with proton irradiated samples are lower than the values after neutron irradiation. The reason for this is a larger acceptor removal constant and smaller introduction rate of deep acceptors. The latter is expected in oxygen rich silicon [18. Given a large uncertainty on the fluence received by samples irradiated at LANSCE the value of $g_{\mathrm{C}}$ was estimated to be in the range of $0.003-0.01 \mathrm{~cm}^{-1}$. For the same reason the acceptor removal parameters could not be estimated. A very low value of $N_{\text {eff }}$ measured with the LANSCE irradiated $1000 \Omega \mathrm{cm}$ sample could be the consequence of acceptor 


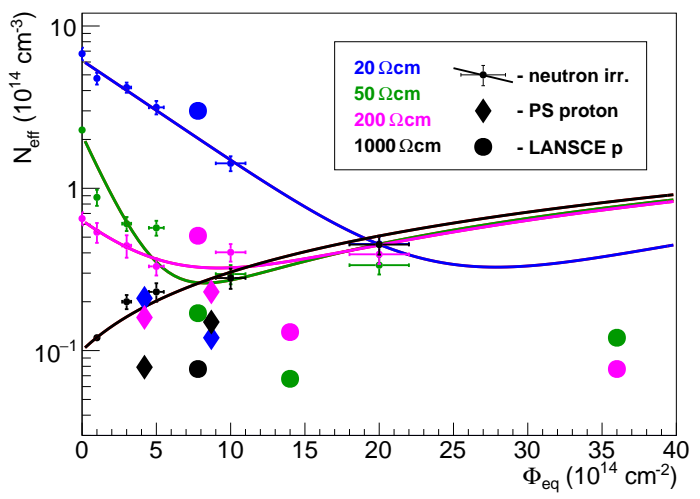

Figure 3: Dependence of effective space charge concentration $N_{\text {eff }}$ on fluence for wafers of different initial resistivity. Data for neutron irradiated samples is fitted with the function 2 which describes acceptor removal.

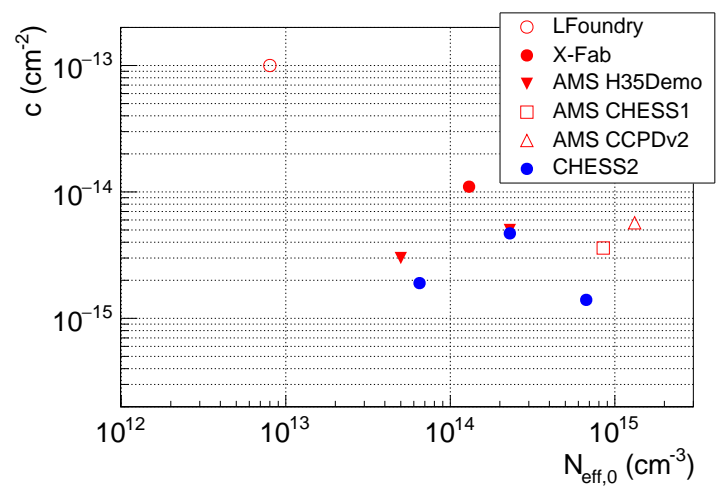

Figure 4: Comparison of measured acceptor removal constants with other HV-CMOS samples: LFoundry [7, X-Fab 8], AMS H35Demo [9], AMS CHESS1 [10] and AMS CCPDv2 [10]. AMS H35Demo and CHESS2 are produced by the same manufacturer in the same CMOS process and have matching values of $c$. 


\begin{tabular}{l|c|c|c|c|} 
& $20 \Omega \mathrm{cm}$ & $50 \Omega \mathrm{cm}$ & $200 \Omega \mathrm{cm}$ & $1000 \Omega \mathrm{cm}$ \\
\hline$N_{\text {eff0 }}\left(10^{14} \mathrm{~cm}^{-3}\right)$ & $6.1 \pm 0.4$ & $2.1 \pm 0.1$ & $0.63 \pm 0.06$ & $0.010 \pm 0.003$ \\
\hline$N_{\mathrm{c}} / N_{\text {eff0 }}$ & $1.06 \pm 0.02$ & $0.98 \pm 0.02$ & $0.95 \pm 0.28$ & $\mathrm{n} / \mathrm{a}$ \\
\hline$c\left(10^{-14} \mathrm{~cm}^{2}\right)$ & $0.14 \pm 0.03$ & $0.47 \pm 0.06$ & $0.19 \pm 0.08$ & $\mathrm{n} / \mathrm{a}$ \\
\hline$g_{\mathrm{C}}\left(\mathrm{cm}^{-1}\right)$ & $0.02($ fixed $)$ & $0.02($ fixed $)$ & $0.02($ fixed $)$ & $0.020 \pm 0.003$ \\
\hline
\end{tabular}

Table 2: Fitted acceptor removal parameters for neutron irradiated samples. confirm this.

\section{Charge collection measurements}

For measurements of charge collection the sample was mounted between two collimators with ${ }^{90} \mathrm{Sr}$ source on one side and a scintillator coupled to a photomultiplier for triggering on the other side. The large pixel array was used for this measurement to ensure that all particles passing the collimators deposit their energy in the active region. Signal from a charge sensitive amplifier with a $25 \mathrm{~ns}$ shaping time was recorded by a digital oscilloscope. More details about the setup can be found in 20. A convolution of Landau and Gauss function was fitted to the measured spectrum of collected charge. Because of a small signal to noise ratio the average value of the spectrum was used instead of the most probable value as the measure of collected charge. The ratio between the average and the most probable signal is approximately 1.25 for ${ }^{90} \mathrm{Sr}[20]$.

Collected charge was measured for different bias voltages up to the electrical breakdown in the range of $80-120 \mathrm{~V}$ where noise increases significantly. Mean collected charge at $100 \mathrm{~V}$ is taken as a figure of merit. For samples where breakdown occurs below $100 \mathrm{~V}$ an extrapolation is made. The results are shown in Figure 5. There is a significant drop of collected charge after the first neutron irradiation step. This is explained by charge collection from the undepleted bulk 145 by diffusion, whose contribution quickly diminishes by irradiation. For the 20, 50 and $200 \Omega \mathrm{cm}$ samples the collected charge increases again at higher fluences 
due to acceptor removal. For 200 and $1000 \Omega \mathrm{cm}$ samples a collected charge above 2000 electrons is measured in the entire fluence range. The $200 \Omega \mathrm{cm}$ sample also shows a relatively small variation of charge with fluence, ranging between 2000 and 3000 electrons after neutron irradiation. As already indicated by Edge-TCT the collected charge in proton irradiated samples is higher due to a larger depletion region. In the $1000 \Omega \mathrm{cm}$ sample irradiated at LANSCE to $7.8 \times 10^{14} \mathrm{n}_{\mathrm{eq}} \mathrm{cm}^{-2}$ (probably overestimated) a charge of 16000 electrons is measured which is more than expected in an unirradiated sample using an extrapolation from Figure 3. This is an indication of acceptor removal also in the highest resistivity substrate.

Measurements with Edge-TCT have shown depletion depths in the range of 30-150 $\mu \mathrm{m}$, which would translate to 3000-15000 electrons of average deposited charge assuming an average charge deposition of $100 \mathrm{e}-\mathrm{h}$ pairs $/ \mu \mathrm{m}$ [20. However, the measured charge is about a factor of two lower from this figure. A possible source of the mismatching is a low charge collection efficiency due to top biasing configuration described in Section 2. The shape of the electric field is such that charge carriers have to drift through a low electric field region at the end of the depletion zone in the substrate, where trapping probability is high after irradiation. As a result a substantial amount of charge carriers gets trapped and does not contribute the full signal, resulting in a reduced charge collection efficiency. This particularly impacts charge collection in pad geometry, where the weighting field is relatively uniform. In case of pixel or strip geometry where the largest weighting field is close to the implants this effect would not affect charge collection so much. A mitigation of this effect could be achieved by biasing the detector from the back, but this approach requires back plane processing 21 .

\section{Conclusions}

Charge collection in neutron and proton irradiated CHESS2 HV-CMOS chips was evaluated by Edge-TCT and minimum ionising electrons from ${ }^{90} \mathrm{Sr}$. 


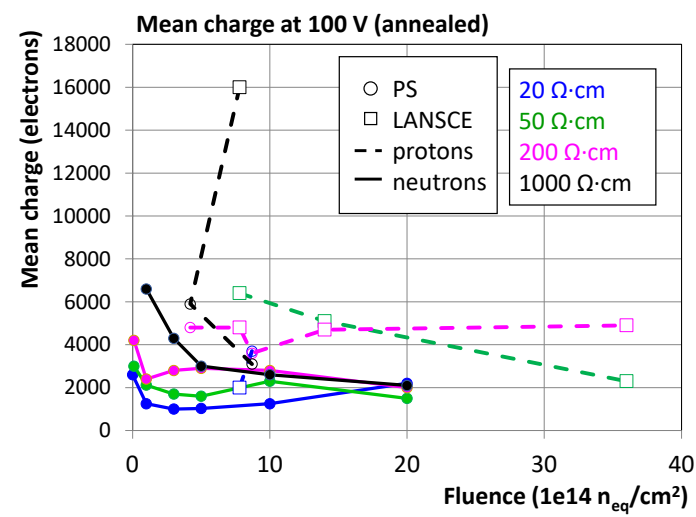

Figure 5: Collected charge in irradiated samples measured with ${ }^{90} \mathrm{Sr}$. Mean charge is shown due to a low signal to noise ratio of the measurement.

Samples of four different initial resistivities in the range of $20-1000 \Omega \mathrm{cm}$ were irradiated up to a fluence of $2 \times 10^{15} \mathrm{n}_{\text {eq }} \mathrm{cm}^{-2}$ with neutrons and $3.6 \times 10^{15} \mathrm{n}_{\mathrm{eq}} \mathrm{cm}^{-2}$ with protons. With Edge-TCT depletion depths of $>70 \mu \mathrm{m}$ at $100 \mathrm{~V}$ were measured in the 200 and $1000 \Omega \mathrm{cm}$ samples at all fluences. The measured depleted depth in $20-200 \Omega \mathrm{cm}$ samples increased after irradiation due to the the effective removal of initial acceptors. In $1000 \Omega \mathrm{cm}$ the acceptor removal was not directly observed and studies at lower fluences would be needed to measure this effect. It was found that acceptor removal constant is larger with protons, i.e. the removal is finished at a lower fluence than with neutrons, and that the stable damage introduction rate is lower. This results in larger depletion depths which mostly exceed $100 \mu \mathrm{m}$ in all samples.

The measurements of charge collection with ${ }^{90} \mathrm{Sr}$ confirm that collected charge increases after irradiation in $20-200 \Omega \mathrm{cm}$ samples due to acceptor removal. The highest charge was measured with the 200 and $1000 \Omega \mathrm{cm}$ samples, where the average collected charge at $100 \mathrm{~V}$ exceeds 2000 electrons at all neutron fluences. In proton irradiated samples the collected charge is larger due to larger depletion depths.

The measured charge is less than the amount of charge deposited in depletion region estimated with Edge-TCT. A similar observation was made in 21], where 
trapping in the low electric field region. This effect is smaller in segmented detectors or in detectors with a processed back plane.

\section{Acknowledgements}

The authors would like to thank the crew at the TRIGA reactor in Ljubl-

Horizon 2020 Research and Innovation programme under Grant Agreement no. 654168.

\section{References}

[1] G. Apollinari, et al., High-Luminosity Large Hadron Collider (HL-LHC):

[4] D.-L. Pohl, et al., Radiation hard pixel sensors using high-resistive wafers in a 150 nm CMOS processing line, JINST 12 (2017) P06020.

[5] H. Pernegger, et al., First tests of a novel radiation hard CMOS sensor process for Depleted Monolithic Active Pixel Sensors, JINST 12 (2017) 220 Preliminary Design Report, CERN Yellow Reports: Monographs, CERN, Geneva, 2015.

[2] G. Aad, et al., ATLAS pixel detector electronics and sensors, JINST 3 (2008) P07007.

[3] ATLAS Phase-II Upgrade Scoping Document, Technical Report CERNLHCC-2015-020. LHCC-G-166, CERN, Geneva, 2015.

P06008. 
[6] M. F. Garcia, C. Gallrapp, M. Moll, D. Muenstermann, Radiation hardness studies of neutron irradiated CMOS sensors fabricated in the AMS H18 high voltage process, JINST 11 (2016) P02016.

[7] I. Mandić, et al., Neutron irradiation test of depleted CMOS pixel detector prototypes, JINST 12 (2017) P02021.

[8] B. Hiti, et al., Charge collection properties in an irradiated pixel sensor built in a thick-film HV-SOI process, JINST 12 (2017) P10020.

[9] E. Cavallaro, et al., Studies of irradiated AMS H35 CMOS detectors for the ATLAS tracker upgrade, JINST 12 (2017) C01074.

[10] A. Affolder, et al., Charge collection studies in irradiated HV-CMOS particle detectors, JINST 11 (2016) P04007.

[11] V. Fadeyev, et al., Investigation of HV/HR-CMOS technology for the ATLAS Phase-II Strip Tracker Upgrade, NIM A 831 (2016) 189 - 196. Proceedings of the 10th International Hiroshima Symposium on the Development and Application of Semiconductor Tracking Detectors.

[12] R. Wunstorf, et al., Investigations of donor and acceptor removal and long term annealing in silicon with different boron/phosphorus ratios, NIM A 377 (1996) 228 - 233. Proceedings of the Seventh European Symposium on Semiconductor.

[13] H. Grabas, Chess2 front end readout of the multi-segmented HV CMOS sensors, in: 10th International Meeting on Front-End Electronics, Warszaw, Poland, 2016.

[14] L. Snoj, G. Žerovnik, A. Trkov, Computational analysis of irradiation facilities at the JSI TRIGA reactor, Applied Radiation and Isotopes 70 (2012) $483-488$.

[15] F. Ravotti, et al., IRRAD: The New $24 \mathrm{GeV} / \mathrm{c}$ Proton Irradiation Facility at CERN (2015) 182-187. Proceedings of AccApp'15. 
[16] G. Kramberger, et al., Investigation of Irradiated Silicon Detectors by Edge-TCT, IEEE Transactions on Nuclear Science 57 (2010) 2294-2302.

[17] G. Kramberger, et al., Radiation effects in low gain avalanche detectors after hadron irradiations, JINST 10 (2015) P07006.

[18] G. Lindström, et al., Radiation hard silicon detectors-developments by the RD48 (ROSE) collaboration, NIM A 466 (2001) 308 - 326. 4th Int. Symp. on Development and Application of Semiconductor Tracking Detectors.

[19] V. Cindro, et al., Radiation damage in p-type silicon irradiated with neutrons and protons, NIM A 599 (2009) $60-65$.

[20] G. Kramberger, et al., Charge collection properties of heavily irradiated epitaxial silicon detectors, NIM A 554 (2005) $212-219$.

[21] I. Mandić, et al., Charge collection properties of irradiated depleted CMOS pixel test structures, arXiv:1801.03671 (2018). 